\title{
Advanced electrodynamic mechanisms for the optical control of light
}

\section{David Bradshaw and Jamie Leeder}

Nonlinear quantum mechanical interactions between light and matter could lead to all-optical switching and transistor action for opticalcomputing platforms.

Among the fundamental interactions between light and matter, many are achieved via engagements that are linear in nature (i.e., variations in the electric field of the light produce a directly proportional response in the material). Linear optical processes, such as single-photon absorption, have been studied at length and feature prominently in technological applications, including UV-visible spectroscopy and photothermal optical microscopy. Recently, interest has grown in the development of optical mechanisms that enable more rapid absorption of light.

To this end, we have developed a scheme involving the input of an off-resonant probe beam that enables highly localized and essentially instant control over absorption events. Under these circumstances, the response of the material is no longer directly proportional to the variation in the electric field of the light and optical nonlinearity is conferred to the system.

This novel development, which enables light to be controlled by light, can be comprehensively described using quantum electrodynamics. In this theoretical framework, the rate equations for any photophysical process are developed from quantum amplitudes. These amplitudes are cast as an infinite series of terms, the order of which relates to the number of light-matter interactions. For example, a first-order term characterizes singlephoton absorption, which features a single light-matter interaction. To simulate the application of an off-resonant probe beam (i.e., an optical input with a wavelength in the transparent region of the irradiated material), we consider a nonlinear contribution to single-photon absorption, which corresponds to a third-order term of the quantum amplitude: see Figure 1. Because the throughput light undergoes forward elastic scattering (a)

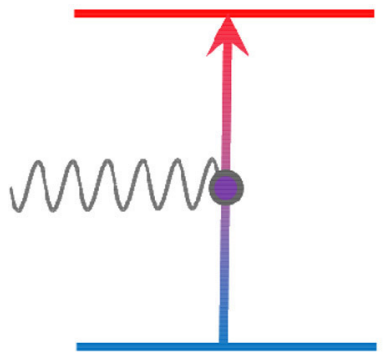

(b)

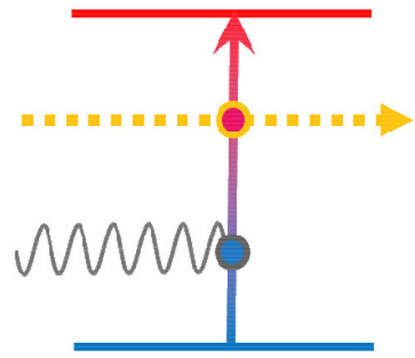

Figure 1. Energy level representation for (a) one-photon absorption and (b) a nonlinear coupling process involving the off-resonant beam. Red and blue horizontal lines represent electronic states of a light-absorbing material. The gray-wavy and yellow-dashed lines represent resonant and off-resonant laser inputs, respectively. The gray circle specifies one-photon absorption. Forward elastic scattering, which involves two light-matter interactions, is indicated by a yellow circle.

and therefore emerges unchanged, the mechanism involves no net absorption of the probe beam. Detailed quantum analysis ${ }^{1,2}$ shows that the efficiency of one-photon absorption can be enhanced by $\sim 20 \%$ for an input light irradiance of $2 \times 10^{15} \mathrm{Wm}^{-2}$.

We have also theoretically shown that due to a mechanism termed laser-controlled fluorescence, the efficiency of emission may be improved by an off-resonant probe beam. ${ }^{3,4}$ This is another process that undergoes no net probe-beam absorption because the laser frequency does not match the emission energy. This lies in contrast to the method wherein the throughput produces stimulated emission, which represents the basis for the Nobel Prize-winning technique of stimulated emission depletion spectroscopy. ${ }^{5-7}$

The output of a three-level laser that is optically pumped just below threshold for the purpose of stimulated emission may also 


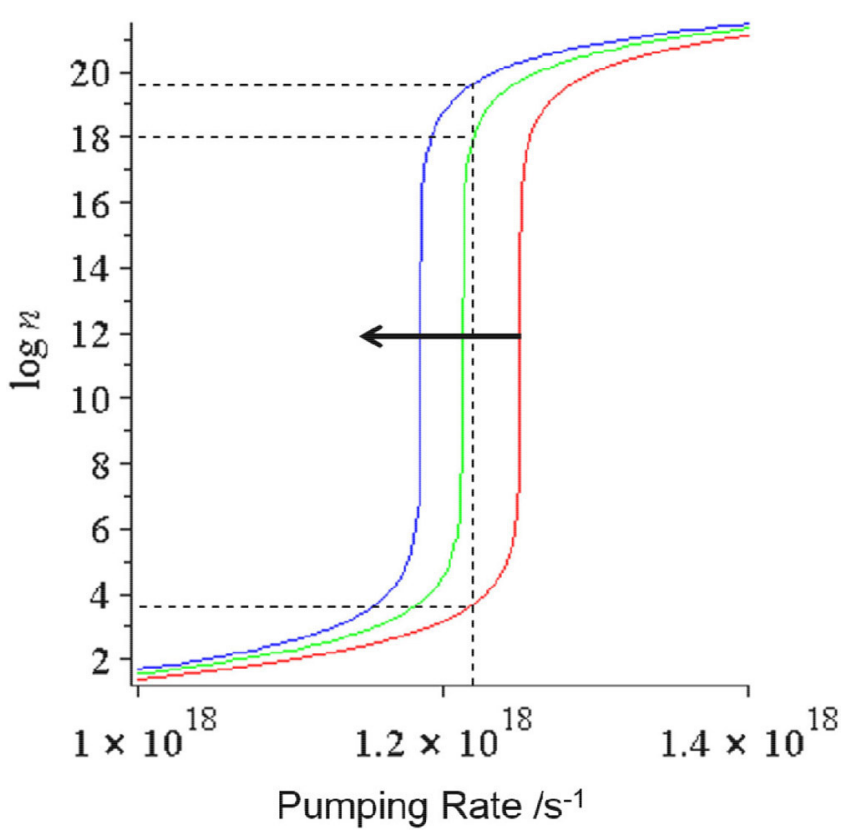

Figure 2. The number of emitted photons (n), plotted as $\log n$, against the pumping rate in the absence (red line) and presence of an offresonant probe beam with an irradiance of $2 \times 10^{11} \mathrm{Wcm}^{-2}$ (green) and $4 \times 10^{11} \mathrm{Wcm}^{-2}$ (blue). The lasing threshold, as indicated by the sudden increase in $n$, reduces with increasing probe-beam intensity. With suitable pumping, a shift to above-threshold operation occurs (upper dotted lines). These plots are produced directly from our equations. ${ }^{8}$

be strongly enhanced by an off-resonant beam. Analysis demonstrates that pulses of light from the off-resonant probe may modify the amplification kinetics of the active medium and thereby enhance laser output: see Figure 2. For a constant pumping rate, the system operates below threshold when the probe beam is absent. Upon the introduction of an off-resonant beam with an irradiance approaching $2 \times 10^{11} \mathrm{Wcm}^{-2}$, the output climbs by approximately 14 orders of magnitude, rising to 16 orders if the input intensity is doubled. This scheme highlights the possibility of all-optical transistor action with respect to the probe beam. ${ }^{8}$

In the case of laser-controlled one-photon absorption, an interesting development arises when we consider a photoactive molecule that cannot undergo a linear engagement (i.e., transitions to the electronic excited state are one-photon forbidden). On application of the off-resonant probe beam, an alternative three-photon-allowed pathway to the excited state (referred to as a 'dark' or metastable state) enables the nonlinear interaction. Because the throughput and absence of the probe beam result in the activation and deactivation of the absorption, respectively, this behavior may provide a basis for an all-optical switch. By extension, the same all-optical principles could be applied to an emissive system in which single-photon emission is forbidden. These principles could also make it possible for data to be written, via laser-controlled absorption, to a molecule containing a dark state, and read using the analogous laser-controlled emission technique. Such a device would be confined to the realm of ultrafast processing, in which a temporary data register of a few nanoseconds is sufficiently long-lived.

In summary, the ability to confer optical nonlinearity upon photoactive materials has been shown to produce new mechanistic features that lead to a variety of potential applications, including all-optical switching and transistor action for ultrafast optical computing. ${ }^{9}$ The theoretical framework we have presented acts as a basis for modeling actual materials in the search for the most efficient system, and invites experimental exploration with a view to the production of novel optical devices.

Research on quantum electrodynamics at the University of East Anglia is supported by the Engineering and Physical Sciences Research Council (EPSRC) and the UK's Leverhulme Trust.

\section{Author Information}

\section{David Bradshaw and Jamie Leeder}

University of East Anglia

Norwich, United Kingdom

\section{References}

1. D. S. Bradshaw and D. L. Andrews, Laser-modified one- and two-photon absorption: expanding the scope of optical nonlinearity, Phys. Rev. A 88, p. 033807, 2013.

2. D. L. Andrews and D. S. Bradshaw, Optically tailored access to metastable electronic states, Chem. Phys. Lett. 590, pp. 235-238, 2013.

3. D. S. Bradshaw and D. L. Andrews, All-optical control of molecular fluorescence, Phys. Rev. A 81, p. 013424, 2010.

4. J. M. Leeder, D. S. Bradshaw, and D. L. Andrews, Laser-controlled fluorescence in two-level systems, J. Phys. Chem. B 115, pp. 5227-5233, 2011.

5. B. Harke, J. Keller, C. K. Ullal, V. Westphal, A. Schöenle, and S. W. Hell, Resolution scaling in STED microscopy, Opt. Express 16, pp. 4154-4162, 2008.

6. S. W. Hell, Microscopy and its focal switch, Nat. Methods 6, pp. 24-32, 2009.

7. E. Rittweger, K. Y. Han, S. E. Irvine, C. Eggeling, and S. W. Hell, STED microscopy reveals crystal colour centres with nanometric resolution, Nat. Photon. 3, pp. 144-147, 2009.

8. D. L. Andrews and D. S. Bradshaw, Off-resonant activation of optical emission, Opt. Commun. 283, pp. 4365-4367, 2010.

9. D. L. Andrews, J. M. Leeder, and D. S. Bradshaw, Advanced electrodynamic mechanisms for the nanoscale control of light by light, Proc. SPIE 9546, p. 95460O, 2015. doi:10.1117/12.2190191 